\title{
Radiopure tungstate and molybdate crystal scintillators for double beta decay experiments
}

\author{
F.A. Danevich \\ Institute for Nuclear Research, 03028 Kyiv, Ukraine \\ CSNSM, Univ. Paris-Sud, CNRS/IN2P3, Université Paris-Saclay, 91405 Orsay, France
}

Crystal scintillators are very promising detectors to investigate double beta decay of atomic nuclei. Recent achievements in development and application of tungstate and molybdate crystal scintillators as well as prospects for the next generation double beta decay experiments are discussed.

Keywords: Double beta decay; Tungstate and Molybdate crystal scintillators; Low counting experiment

\section{Introduction}

Double beta $(2 \beta)$ decay of atomic nuclei is the rarest nuclear process observed by human being. Despite the two neutrino mode $(2 \nu)$ of the decay is allowed by all the known laws, its probability is extremely low. After the great efforts of experimentalists over seventy years, by utilizing different methods: geochemical, radiochemical, direct counting, the decay is observed in eleven nuclei with the half-lives in the range $T_{1 / 2} \sim 10^{18}-10^{24} \mathrm{yr}[1,2,3]$.

The neutrinoless mode of double beta decay $(0 \nu 2 \beta)$ is forbidden in the Standard Model of particle physics since the decay breaks the lepton number and calls for the neutrinos to be Majorana particles with nonzero mass [4]. The process is considered as one of the most powerful tools to study properties of neutrino and weak interaction, and to test the Standard Model of particle physics [5, 6, 17, 8, 9, 10].

In contrast to the $2 \nu 2 \beta^{-}$decay that is already observed, even the most sensitive experiments give only half-life limits at level of $T_{1 / 2}>10^{24}-10^{26} \mathrm{yr}$ on the $0 \nu$ mode of decay (we refer reader to the reviews [11, 12, 13, 14, 15, 9] and to the recent experimental searches [16, 17, 18, 19, 20]). The experiments set limits on the effective Majorana neutrino mass at the level of $\left\langle m_{\nu}\right\rangle \sim 0.1$ $-1 \mathrm{eV}$. The range is due to the ambiguity of the theoretical calculations of nuclear matrix elements of $0 \nu 2 \beta^{-}$decay [21].

The sensitivity of the experiments to search for the double electron capture $(2 \varepsilon)$, electron capture with positron emission $\left(\varepsilon \beta^{+}\right)$, and double positron emission $\left(2 \beta^{+}\right)$is much lower. In contrast to the $2 \beta^{-}$decay, even the allowed two neutrino mode of these processes has not yet been detected clearly (see, e.g, reviews [1, 22]). However, development of experimental techniques to search for the processes is requested, taking into account a capability to refine the possible contribution of the right-handed currents to the $0 \nu 2 \beta^{-}$decay rate if the decay will be observed [23]. In addition, a resonant enhancement of the $0 \nu 2 \varepsilon$ process can increase the decay rate by several orders of magnitude in the case of coincidence between the released energy and the energy of an excited state of the daughter nucleus [24, 25, 26, 27, 28, 29, 30].

The goal of the next generation $2 \beta^{-}$experiments is to explore the inverted hierarchy of 
the neutrino mass (the effective Majorana mass of neutrino $\left\langle m_{\nu}\right\rangle \approx 0.02-0.05 \mathrm{eV}$ ). Such a sensitivity can be reached in experiments able to detect an extremely low level of $0 \nu 2 \beta^{-}$ activity with the half-lives in the range $T_{1 / 2} \sim 10^{26}-10^{27} \mathrm{yr}$. The realization of such ambitious plans requires construction of detectors containing a large number of $2 \beta^{-}$active nuclei $\left(10^{27}-\right.$ $10^{28}$ nuclei, $\sim 10^{3}-10^{4}$ moles of isotope of interest), extremely low (ideally zero) radioactive background, high detection efficiency and ability to distinguish the $0 \nu 2 \beta^{-}$decay events, in particular, as high as possible energy resolution. It should be stressed that a low energy resolution is acceptable as far as an experiment set a limit on the process searched for. However, in a case of an effect evidence the poor energy resolution become to be a controversial factor in the effect interpretation.

Taking into account an extremely low decay probability and the ambiguity of the theoretical calculations of the nuclear matrix elements, the experimental program should include several nuclei candidates. First of all, confirmation in different experiments will be naturally requested in a case of a positive evidence of the $0 \nu 2 \beta^{-}$decay in one nucleus. Moreover, data on $0 \nu 2 \beta^{-}$ decay rate in several nuclei will be useful to adjust theoretical approaches to calculate the nuclear matrix elements [31].

Therefore, choice of candidate nuclei is determined by the scale of experiments (several hundred kilograms of isotope of interest), extreme requirements to background and possibility of calorimetric experiments ("source = detector") to provide high detection efficiency and energy resolution. In this paper we would like to argue that scintillation detectors, especially low temperature scintillating bolometers, are very promising to realize large scale high sensitivity $0 \nu 2 \beta^{-}$decay experiments with several nuclei.

\section{Scintillation detectors in double beta decay experi- ments}

Crystal scintillators possess certain advantages for double beta decay experiments thanks to presence of the element of interest in the crystal scintillator compound, that provides a high detection efficiency for the effect searched for. Cadmium and zinc tungstates, zinc selenide crystal scintillators were studied as detector candidates for double beta experiments in [32], lead molybdate was proposed to search for $2 \beta^{-}$decay of ${ }^{100}$ Mo in [33].

The most sensitive $2 \beta$ experiments with crystal scintillators are reported in Table 1. We would like to repeat that the current sensitivity of the experiments to search for $2 \varepsilon, \varepsilon \beta^{+}$and $2 \beta^{+}$decays is a few orders of magnitude lower than that of the $2 \beta^{-}$experiments. Therefore, the listen limits for the $2 \varepsilon$ and $\varepsilon \beta^{+}$decays correspond broadly to the level of the most sensitive investigations in the field. It should be stressed that the half-life values and limits for the $2 \beta^{-}$ decay of ${ }^{100} \mathrm{Mo}$ are very preliminary results of the R\&Ds in progress aiming at construction of large scale cryogenic experiments to search for $0 \nu 2 \beta^{-}$decay in ${ }^{100}$ Mo with a sensitivity a few orders of magnitude higher than that the reported in Table 1 (the projects will be discussed in Section 4).

Characteristics of the $2 \beta$ isotopes present in the already developed tungstate and molybdate crystal scintillators are listed in Table 2 . 
Table 1: The most sensitive $2 \beta$ experiments with crystal scintillators.

\begin{tabular}{|l|l|l|l|}
\hline $2 \beta$ transition & Scintillator & Main results: half-life (channels) & Ref. \\
\hline${ }^{40} \mathrm{Ca} \rightarrow{ }^{40} \mathrm{Ar}$ & $\mathrm{CaWO}_{4}$ & $\begin{array}{l}\geq 9.9 \times 10^{21} \mathrm{yr}(2 \nu 2 K) \\
\geq 1.4 \times 10^{22} \mathrm{yr}(0 \nu 2 \varepsilon)\end{array}$ & {$[34]$} \\
& & $\geq 5.8 \times 10^{22} \mathrm{yr}\left(0 \nu 2 \beta^{-}\right)$ & {$[34]$} \\
\hline${ }^{48} \mathrm{Ca} \rightarrow{ }^{48} \mathrm{Ti}$ & $\mathrm{CaF}_{2}(\mathrm{Eu})$ & {$[35]$} \\
\hline${ }^{64} \mathrm{Zn} \rightarrow{ }^{64} \mathrm{Ni}$ & $\mathrm{ZnWO}_{4}$ & $\geq 1.1 \times 10^{19} \mathrm{yr}(2 \nu 2 K)$ & {$[36]$} \\
& & $\geq 9.4 \times 10^{20} \mathrm{yr}\left(2 \nu \varepsilon \beta^{+}\right)$ & {$[36]$} \\
\hline${ }^{100} \mathrm{Mo} \rightarrow{ }^{100} \mathrm{Ru}$ & $\mathrm{ZnMoO}_{4}$ & $=[7.15 \pm 0.37($ stat. $) \pm 0.66($ syst. $)] \times 10^{18} \mathrm{yr}\left(2 \nu 2 \beta^{-}\right)$ & {$[37]$} \\
& $\mathrm{Li}_{2}^{100} \mathrm{MoO}_{4}$ & $=[6.92 \pm 0.06($ stat. $) \pm 0.36($ syst. $)] \times 10^{18} \mathrm{yr}\left(2 \nu 2 \beta^{-}\right)$ & {$[38]$} \\
& $\mathrm{Zn}^{100} \mathrm{MoO}_{4}$ & $\geq 2.6 \times 10^{22} \mathrm{yr}\left(0 \nu 2 \beta^{-}\right)$ & {$[38$} \\
& ${ }^{48 d e p l} \mathrm{Ca}^{100} \mathrm{MoO}_{4}$ & $\geq 4.0 \times 10^{21} \mathrm{yr}\left(0 \nu 2 \beta^{-}\right)$ & {$[39]$} \\
\hline${ }^{106} \mathrm{Cd} \rightarrow{ }^{106} \mathrm{Pd}$ & ${ }^{106} \mathrm{CdWO}_{4}$ & $\geq 1.1 \times 10^{21} \mathrm{yr}\left(2 \nu \varepsilon \beta^{+}\right)$ & {$[40]$} \\
& & $\geq 2.2 \times 10^{21} \mathrm{yr}\left(0 \nu \varepsilon \beta^{+}\right)$ & {$[41]$} \\
\hline${ }^{116} \mathrm{Cd} \rightarrow{ }^{116} \mathrm{Sn}$ & ${ }^{116} \mathrm{CdWO}_{4}$ & $=[2.69 \pm 0.02($ stat. $) \pm 0.14($ syst. $)] \times 10^{19} \mathrm{yr}\left(2 \nu 2 \beta^{-}\right)$ & {$[42]$} \\
& & $\geq 2.4 \times 10^{23} \mathrm{yr}\left(0 \nu 2 \beta^{-}\right)$ & {$[42]$} \\
\hline${ }^{130} \mathrm{Ba} \rightarrow{ }^{130} \mathrm{Xe}$ & $\mathrm{BaF}_{2}$ & $\geq 2.0 \times 10^{17} \mathrm{yr}\left(0 \nu \varepsilon \beta^{+}\right)$ & {$[43]$} \\
\hline${ }^{136} \mathrm{Ce} \rightarrow{ }^{136} \mathrm{Ba}$ & $\mathrm{CeCl}_{3}$ & $\geq 3.2 \times 10^{16} \mathrm{yr}(2 \nu 2 K)$ & {$[44]$} \\
\hline${ }^{160} \mathrm{Gd} \rightarrow{ }^{160} \mathrm{Dy}$ & $\mathrm{Gd}_{2} \mathrm{SiO}_{5}(\mathrm{Ce})$ & $\geq 1.3 \times 10^{21} \mathrm{yr}\left(0 \nu 2 \beta^{-}\right)$ & {$[45]$} \\
\hline${ }^{180} \mathrm{~W} \rightarrow{ }^{180} \mathrm{Hf}$ & $\mathrm{CaWO}_{4}$ & $\geq 3.1 \times 10^{19} \mathrm{yr}(2 \nu 2 K)$ & {$[34]$} \\
& & $\geq 9.4 \times 10^{18} \mathrm{yr}(2 \nu 2 \varepsilon)$ & {$[34]$} \\
\hline
\end{tabular}


Table 2: Characteristics of $2 \beta$ isotopes present in tungstate and molybdate crystal scintillators.

\begin{tabular}{|l|l|l|l|}
\hline $2 \beta$ transition & $Q_{2 \beta}(\mathrm{keV})[46]$ & Isotopic abundance (\%) [47] & Decay channel \\
\hline${ }^{40} \mathrm{Ca} \rightarrow{ }^{40} \mathrm{Ar}$ & $193.51(2)$ & $96.941(156)$ & $2 \varepsilon$ \\
\hline${ }^{46} \mathrm{Ca} \rightarrow{ }^{46} \mathrm{Ti}$ & $988.4(2.2)$ & $0.004(3)$ & $2 \beta^{-}$ \\
\hline${ }^{48} \mathrm{Ca} \rightarrow{ }^{48} \mathrm{Ti}$ & $4268.08(8)$ & $0.187(21)$ & $2 \beta^{-}$ \\
\hline${ }^{64} \mathrm{Zn} \rightarrow{ }^{64} \mathrm{Ni}$ & $1094.9(7)$ & $49.17(75)$ & $2 \varepsilon, \varepsilon \beta^{+}$ \\
\hline${ }^{70} \mathrm{Zn} \rightarrow{ }^{70} \mathrm{Ge}$ & $997.1(2.1)$ & $0.61(10)$ & $2 \beta^{-}$ \\
\hline${ }^{84} \mathrm{Sr} \rightarrow{ }^{84} \mathrm{Kr}$ & $1789.8(1.2)$ & $0.56(2)$ & $2 \varepsilon, \varepsilon \beta^{+}$ \\
\hline${ }^{92} \mathrm{Mo} \rightarrow{ }^{92} \mathrm{Zr}$ & $1650.45(19)$ & $14.649(106)$ & $2 \varepsilon, \varepsilon \beta^{+}$ \\
\hline${ }^{98} \mathrm{Mo} \rightarrow{ }^{98} \mathrm{Ru}$ & $109(6)$ & $24.292(80)$ & $2 \beta^{-}$ \\
\hline${ }^{100} \mathrm{Mo} \rightarrow{ }^{100} \mathrm{Ru}$ & $3034.36(17)$ & $9.744(65)$ & $2 \beta^{-}$ \\
\hline${ }^{106} \mathrm{Cd} \rightarrow{ }^{106} \mathrm{Pd}$ & $2775.39(10)$ & $1.245(22)$ & $2 \varepsilon, \varepsilon \beta^{+}, 2 \beta^{+}$ \\
\hline${ }^{108} \mathrm{Cd} \rightarrow{ }^{108} \mathrm{Pd}$ & $271.8(8)$ & $0.888(11)$ & $2 \varepsilon$ \\
\hline${ }^{114} \mathrm{Cd} \rightarrow{ }^{114} \mathrm{Sn}$ & $544.79(28)$ & $28.754(81)$ & $2 \beta^{-}$ \\
\hline${ }^{116} \mathrm{Cd} \rightarrow{ }^{116} \mathrm{Sn}$ & $2813.49(13)$ & $7.512(54)$ & $2 \beta^{-}$ \\
\hline${ }^{180} \mathrm{~W} \rightarrow{ }^{180} \mathrm{Hf}$ & $143.23(28)$ & $0.12(1)$ & $2 \beta^{-}$ \\
\hline${ }^{186} \mathrm{~W} \rightarrow{ }^{186} \mathrm{Os}$ & $491.4(1.2)$ & $28.43(19)$ & \\
\hline
\end{tabular}

\section{Requirements to crystal scintillators}

Sensitivity of a $0 \nu 2 \beta^{-}$counting experiment can be expressed by the following formula [48]:

$$
\lim T_{1 / 2} \sim \varepsilon \cdot \delta \sqrt{\frac{m \cdot t}{R \cdot B G}},
$$

where $\varepsilon$ is detection efficiency of the $0 \nu 2 \beta^{-}$effect, $\delta$ is the concentration of the isotope of interest, $t$ is the measurement time, $m$ is the mass, $R$ is the energy resolution and $B G$ is the background per unit mass, time and energy. In a case of very low background ("zero background experiment") the sensitivity is proportional to the exposure of experiment $m \cdot t$ :

$$
\lim T_{1 / 2} \sim \varepsilon \cdot \delta \frac{m \cdot t}{\lim S}
$$

where $\lim S$ is number of events that can be excluded at a given confidence level. E.g., according to the Feldman and Cousins procedure [49] $\lim S=2.44,3.28$ and 3.94 at $90 \%$ confidence level (C.L.) in the case of detected 0, 1 and 2 events, respectively.

From the formulas 1 and 2 one can immediately see the advantage of scintillation $0 \nu 2 \beta^{-}$ counting experiment: a high detection efficiency $\varepsilon$ (approaching to $\approx 100 \%$ with increase of the crystal scintillator volume and density) thanks to presence of the element of interest. $\delta$ cannot be $100 \%$ in a crystal scintillator (as, e.g., in the high purity germanium detectors used in the ${ }^{76} \mathrm{Ge}$ experiments). Nevertheless, typically $\delta$ is rather high in tungstate and molybdate crystals. The concentration can be increased by production of crystal scintillators from isotopically enriched materials (see Section 3.2). 
The main properties of tungstate and molybdate crystal scintillators that are interesting for $2 \beta$ experiments are reported in Table 3 . 
Table 3: Properties of tungstate and molybdate crystal scintillators. Wavelength of scintillation emission maximum is denoted as $\lambda_{\max }$; FWHM is full width at half of maximum; $F O M$ is factor of merit of particle discrimination capability (see formula 3); FOM for room temperature scintillators is given for $\approx 5 \mathrm{MeV} \alpha$ particles and $\approx 1 \mathrm{MeV} \gamma$ quanta, while for milli-Kelvin measurements $F O M$ is presented mainly for energy of $\alpha$ particles and $\gamma$ quanta above $2.5 \mathrm{MeV}$.

\begin{tabular}{|c|c|c|c|c|c|c|c|c|c|}
\hline \multirow[t]{2}{*}{ Scintillator } & \multirow{2}{*}{$\begin{array}{l}\text { Density } \\
\left(\mathrm{g} / \mathrm{cm}^{3}\right)\end{array}$} & \multirow{2}{*}{$\begin{array}{l}\text { Melting } \\
\text { point }\left({ }^{\circ} \mathrm{C}\right)\end{array}$} & \multirow{2}{*}{$\begin{array}{l}\text { Index of } \\
\text { refraction }\end{array}$} & \multirow{2}{*}{$\begin{array}{l}\lambda_{\max } \\
(\mathrm{nm})\end{array}$} & \multicolumn{2}{|c|}{ Energy resolution, FWHM } & \multicolumn{2}{|c|}{ Particle discrimination, $F O M$} & \multirow[t]{2}{*}{ Ref. } \\
\hline & & & & & $\begin{array}{l}\text { Room } \\
\text { temperature, } \\
\text { at } 662 \mathrm{keV}\end{array}$ & $\begin{array}{l}\text { milli-Kelvin, } \\
\text { at } 2615 \mathrm{keV}\end{array}$ & $\begin{array}{l}\text { Room } \\
\text { temperature }\end{array}$ & milli-Kelvin & \\
\hline $\mathrm{MgWO}_{4}$ & 5.66 & 1358 & & 470 & $9.1 \%$ & & 5.2 & & 50 \\
\hline $\mathrm{CaWO}_{4}$ & 6.1 & $1570-1650$ & 1.94 & 425 & $6.4 \%$ & $6.7 \mathrm{keV}^{* *}$ & 5.9 & & $51,52,53$ \\
\hline $\mathrm{ZnWO}_{4}$ & 7.8 & 1200 & $2.1-2.2$ & 480 & $8.5 \%$ & $4.7 \mathrm{keV}^{* *}$ & 5.2 & & {$[56,54,55$} \\
\hline $\mathrm{CdWO}_{4}$ & 7.9 & 1325 & $2.2-2.3$ & 480 & $6.8 \%$ & $6.3 \mathrm{keV}$ & 5.8 & 15 & [57, [58, 59] \\
\hline $\mathrm{PbWO}_{4}$ & 8.28 & 1123 & 2.2 & 450 & $23 \% *$ & $15.7 \mathrm{keV}$ & $2.4^{*}$ & & $62,60,61$ \\
\hline $\mathrm{Li}_{2} \mathrm{MoO}_{4}$ & 3.05 & $701 \pm 2$ & 1.44 & 590 & & $4-6 \mathrm{keV}$ & & $9-18$ & 63, 64 \\
\hline $\mathrm{CaMoO}_{4}$ & 4.3 & $1445-1480$ & 2.0 & 520 & $10.3 \%$ & $10.9 \mathrm{keV}$ & 2.2 & 7.6 & 65,66 \\
\hline $\mathrm{ZnMoO}_{4}$ & 4.3 & $1003 \pm 5$ & $1.89-1.96$ & 625 & & $4-22 \mathrm{keV}$ & & $8-21$ & $64,67,68$ \\
\hline $\mathrm{SrMoO}_{4}$ & 4.67 & 1380 & & 506 & & & & & 69 \\
\hline $\mathrm{PbMoO}_{4}$ & 6.95 & 1060 & 2.4 & 540 & & & $2^{* * *}$ & & 70 \\
\hline
\end{tabular}

${ }^{*}$ At temperature $-25^{\circ} \mathrm{C}$.

** For $2.31 \mathrm{MeV} \alpha$.

*** At temperature $-196^{\circ} \mathrm{C}$. 
Energy resolution of the convenient scintillation detectors at room temperature is at the level of several \%. Around the tungstate crystals discussed as $2 \beta$ detectors, the highest energy resolution (full width at half of maximum, FWHM, for $\gamma$ quanta of ${ }^{137} \mathrm{Cs}$ with energy $662 \mathrm{keV}$ ) was achieved with $\mathrm{CaWO}_{4}\left(6.4 \%\right.$, [53]), $\mathrm{CdWO}_{4}$ (6.8\%, [57]), $\mathrm{ZnWO}_{4}$ (8.5\%, [54]) and $\mathrm{MgWO}_{4}$ $\left(9.1 \%\right.$, [50]). As for molybdates, only $\mathrm{CaMoO}_{4}$ shows reasonable scintillation efficiency and energy resolution FWHM $=10.3 \%$ 65].

The energy resolution obtained with the scintillators at low temperatures (denoted in the Table 3 as milli-Kelvin temperatures) is significantly higher, that is a great advantage of the bolometric detection technique.

Most of scintillators posses particle-discrimination capability that allows a substantial suppression of background caused by the internal and surface radioactive contamination of the crystal scintillators by $\alpha$ active nuclides, particularly by uranium, radium and thorium. The particle discrimination capability (denoted here as $F O M$, factor of merit) can be determined by the following formula:

$$
F O M=\left|S I_{\alpha}-S I_{\gamma}\right| / \sqrt{\sigma_{\alpha}^{2}+\sigma_{\gamma}^{2}}
$$

where $S I_{\alpha}\left(\sigma_{\alpha}\right)$ and $S I_{\gamma}\left(\sigma_{\gamma}\right)$ are mean values (standard deviations) of Shape Indicator parameters for $\alpha$ particles and $\gamma$ quanta distributions. The $S I$ parameters can be estimated, e.g., by using the optimal filter method (see the application of the method to $\mathrm{CdWO}_{4}$ scintillators in [71, 57]), or by calculation of the mean time of scintillation signal (see, e.g., [60]).

As one can see in Table 3, cryogenic scintillating bolometers possess a much higher particle discrimination capability than that the conventional scintillation detectors. The discrimination can be realized by analysis of heat and scintillation signals from a crystal scintillator (taking into account a much lower scintillation yield for ions 1 , in particular for $\alpha$ particles [73]) or by only thermal pulse profile analysis [74].

The most important source of scintillation detector background, that finally limits a $2 \beta$ experiment sensitivity, is radioactive contamination of crystal scintillators. The issue is discussed in the following section.

\subsection{Radiopurity}

Radioactive contamination of a scintillation material plays a key role in $2 \beta$ experiments that require as low as possible (ideally zero) background counting rate in the region of interest (ROI). Some data on radioactive contamination of tungstate and molybdate crystal scintillators are presented in Table 4 .

\footnotetext{
${ }^{1}$ Zinc selenide scintillators at milli-Kelvin temperature show an opposite property: a higher emission from $\alpha$ particles than that from $\beta$ particles and $\gamma$ quanta $[2]$.
} 
Table 4: Radioactive contamination of tungstate and molybdate crystal scintillators. Radioactive isotopes of tungstate $\left({ }^{180} \mathrm{~W}, \alpha\right.$ active) and molybdate $\left({ }^{100} \mathrm{Mo}, 2 \beta^{-}\right.$ active) scintillators are not specified. The lowest reported limit is presented if no activity of the nuclide was detected. The lowest limit and the biggest value of activity (or the range of values) are given if several samples of the scintillator were studied.

\begin{tabular}{|c|c|c|c|c|c|c|}
\hline \multirow{2}{*}{ Scintillator } & \multicolumn{5}{|c|}{ Radioactive contamination $(\mathrm{mBq} / \mathrm{kg})$} & \multirow[t]{2}{*}{ Reference } \\
\hline & ${ }^{40} \mathrm{~K}$ & ${ }^{226} \mathrm{Ra}$ & ${ }^{228} \mathrm{Th}$ & total $\alpha$ & $\begin{array}{l}\text { Inherent radionuclides } \\
\text { in scintillator compound }\end{array}$ & \\
\hline $\mathrm{MgWO}_{4}$ & $<1.6 \times 10^{3}$ & $<50$ & $<50$ & $5.7(4) \times 10^{3}$ & & [50] \\
\hline $\mathrm{CaWO}_{4}$ & $\leq 12$ & $6-7$ & $<0.2-0.6(2)$ & $400-930$ & ${ }^{48} \mathrm{Ca}\left(2 \beta^{-}\right)$ & 51,75 \\
\hline $\mathrm{ZnWO}_{4}$ & $<0.02$ & $0.002(1)-0.025(6)$ & $0.002(2)-0.018(2)$ & $0.18(3)-2.3(2)$ & & 76 \\
\hline $\mathrm{CdWO}_{4}$ & $<1.7-3.6(2)$ & $<0.007$ & $<0.003-0.008(4)$ & $0.26(4)$ & ${ }^{113} \mathrm{Cd}(\beta),{ }^{113 m} \mathrm{Cd}(\beta),{ }^{116} \mathrm{Cd}\left(2 \beta^{-}\right)$ & [77, 78, 79 \\
\hline${ }^{106} \mathrm{CdWO}_{4}$ & $<1.4$ & $0.012(3)$ & $0.042(4)$ & $2.1(2)$ & ${ }^{113 m} \mathrm{Cd}(\beta),{ }^{113} \mathrm{Cd}(\beta),{ }^{116} \mathrm{Cd}\left(2 \beta^{-}\right)$ & 41 \\
\hline${ }^{116} \mathrm{CdWO}_{4}$ & $\leq 0.9-0.3(1)$ & $\leq 0.004$ & $0.010(3)-0.062(6)$ & $1.4(1)-2.93(2)$ & ${ }^{113 m} \mathrm{Cd}(\beta),{ }^{113} \mathrm{Cd}(\beta),{ }^{116} \mathrm{Cd}\left(2 \beta^{-}\right)$ & $\begin{array}{l}{[80,81,82} \\
{[83,84]}\end{array}$ \\
\hline $\mathrm{PbWO}_{4}$ & & $1.40(4)$ & $0.051(8)^{*}$ & & ${ }^{210} \mathrm{~Pb}$ and daughters $(\beta, \alpha)$ & 61 \\
\hline $\mathrm{Li}_{2} \mathrm{MoO}_{4}$ & $\leq 3.2-62(2)$ & $\leq 0.028-0.13(2)$ & $\leq 0.018$ & & & 64 \\
\hline $\mathrm{Li}_{2}^{100} \mathrm{MoO}_{4}$ & $\leq 3.5$ & $\leq 0.007$ & $\leq 0.006$ & & & 64 \\
\hline $\mathrm{ZnMoO}_{4}$ & & $\leq 0.006$ & $\leq 0.005$ & & & 64 \\
\hline $\mathrm{Zn}^{100} \mathrm{MoO}_{4}$ & & $0.014(3)-0.023(4)$ & $\leq 0.008$ & & & 64 \\
\hline $\mathrm{CaMoO}_{4}$ & $<1$ & $0.13(4)-2.5(5)$ & $0.04(2)-0.42(17)$ & & ${ }^{48} \mathrm{Ca}\left(2 \beta^{-}\right)$ & 65 \\
\hline${ }^{48 d e p l} \mathrm{Ca}^{100} \mathrm{MoO}_{4}$ & & 0.065 & $<0.05$ & & ${ }^{48} \mathrm{Ca}\left(2 \beta^{-}\right)$ & 85 \\
\hline
\end{tabular}

$$
\text { | }
$$

* Activity of ${ }^{232} \mathrm{Th}$. 
The main radioactive contaminations of scintillation materials are naturally occurring radioactive elements, as uranium (consists of isotopes ${ }^{235} \mathrm{U}$ and ${ }^{238} \mathrm{U}$ ), thorium $\left({ }^{232} \mathrm{Th}\right.$ ) with their daughters, and potassium $\left({ }^{40} \mathrm{~K}\right)$. The secular equilibrium of the $\mathrm{U} / \mathrm{Th}$ chains is typically broken in scintillation materials that pass through chemical and physical procedures of purification, synthesis and crystal growth. It means that the activities of ${ }^{238} \mathrm{U},{ }^{230} \mathrm{Th},{ }^{226} \mathrm{Ra},{ }^{210} \mathrm{~Pb}$, and ${ }^{210} \mathrm{Po}$ in the ${ }^{238} \mathrm{U}$ family should be considered separately. Similarly, activities of ${ }^{232} \mathrm{Th},{ }^{228} \mathrm{Ra}$ and ${ }^{228} \mathrm{Th}$ from the ${ }^{232} \mathrm{Th}$ family, and activities of ${ }^{235} \mathrm{U},{ }^{231} \mathrm{~Pa}$ and ${ }^{227} \mathrm{Ac}$ from the ${ }^{235} \mathrm{U}$ family can be different in a sample of scintillator. For instance, the equilibrium of ${ }^{238} \mathrm{U}$ chain is strongly broken in $\mathrm{CaWO}_{4}$ [51].

Some scintillators contain valuable impurities of primordial radioactive isotopes of rareearth elements. As an example we again refer to $\mathrm{CaWO}_{4}$ where $\alpha$ radioactive ${ }^{144} \mathrm{Nd},{ }^{147} \mathrm{Sm}$, and ${ }^{152} \mathrm{Gd}$ were detected at a $\mathrm{mBq} / \mathrm{kg}$ level [51, 52. Anthropogenic ${ }^{90} \mathrm{Sr}-{ }^{90} \mathrm{Y}$ and ${ }^{137} \mathrm{Cs}$ can also be present in scintillators, especially after the Chernobyl (in 1986), and the Fukushima Daiichi (2012) nuclear disasters. 2 .

Radioactive contamination of crystal scintillators is determined substantially by its chemical formula and possibilities of initial materials purification. For instance $\mathrm{CdWO}_{4}$ and $\mathrm{ZnWO}_{4}$ crystals have typically rather low level of internal contamination, while $\mathrm{CaWO}_{4}$ crystals are rather contaminated, mainly by radium (see Table (4). Same dependence of radium activity on presence of calcium is observed in $\mathrm{ZnMoO}_{4}, \mathrm{Li}_{2} \mathrm{MoO}_{4}$ (highly radiopure materials with only limits on ${ }^{226} \mathrm{Ra}$ and ${ }^{228} \mathrm{Th}$ activities on the level of $<0.005 \mathrm{mBq} / \mathrm{kg}$ ) versus $\mathrm{CaMoO}_{4}$ crystals, where activity of ${ }^{226} \mathrm{Ra}$ is still on the level of $\sim 0.1 \mathrm{mBq} / \mathrm{kg}$, despite the rather strong efforts to reduce the contamination. The contamination of calcium containing crystals is due to the chemical similarity of calcium and radium, and due to the certain difficulties to purify calcium with effective physical purification methods, like vacuum distillation.

The main source of scintillation material radioactive contamination is radioactive contamination of the materials used for powder synthesis and then for the crystal growth. Possible effect of ceramic details contamination of the growing apparats on the $\mathrm{ZnWO}_{4}$ crystal scintillators radiopurity was investigated in [76]. There is no indication of tungstate and molybdate crystal scintillators pollution by the growing process (such as platinum contamination of $\mathrm{TeO}_{2}$ crystals grown in platinum crucibles observed by the CUORE collaboration [87]). Nevertheless, possible effects of the radioactive contamination of the components of the growing set-ups on the radiopurity of the crystal scintillators should be addressed in the future developments of highly radiopure crystal scintillators.

Recrystallization can improve significantly the radiopurity level of tungstate and molybdate crystals [88, 64]. Recently the concentration of thorium was reduced by one order of magnitude, down to $0.01 \mathrm{mBq} / \mathrm{kg}\left({ }^{228} \mathrm{Th}\right.$ ), by recrystallization of the ${ }^{116} \mathrm{CdWO}_{4}$ sample No. 3 (Fig. 1 in [82]) by the low-thermal-gradient Czochralski method. The total $\alpha$ activity of uranium and thorium daughters was reduced by a factor $\approx 3$, down to $1.6 \mathrm{mBq} / \mathrm{kg}[84$.

Cosmogenic radionuclides, i.e. created by high energy neutrons (mainly of cosmic rays origin), were observed in tungstate scintillators: ${ }^{65} \mathrm{Zn}$ in $\mathrm{ZnWO}_{4}$ [76, and ${ }^{110 m} \mathrm{Ag}$ in $\mathrm{CdWO}_{4} 83$ ] crystal scintillators on a $\sim 0.1 \mathrm{mBq} / \mathrm{kg}$ level. To reduce cosmogenic activation the production line and transportation of the ready-to-use scintillation elements to the experiment site should be organized in a way providing minimal cosmogenic activation.

Some scintillators have radioactive elements in their chemical formula. Cadmium tungstate

\footnotetext{
${ }^{2}$ E.g., it was quite expectable to find ${ }^{134} \mathrm{Cs}$ and ${ }^{137} \mathrm{Cs}$ in $\mathrm{CsI}(\mathrm{Tl})$ scintillators 86 .
} 
scintillation crystals contain primordial $\beta$ radioactive isotope ${ }^{113} \mathrm{Cd}\left(Q_{\beta}=323.83(27) \mathrm{keV}\right.$, isotopic abundance $\left.12.227(7) \%, T_{1 / 2}=8.04(5) \times 10^{15} \mathrm{yr}\right)$. Beta active ${ }^{210} \mathrm{~Pb}\left(Q_{\beta}=63.5(5)\right.$ $\left.\mathrm{keV}, T_{1 / 2}=22.20(22) \mathrm{yr}\right)$ is usually present in $\mathrm{PbWO}_{4}$ and $\mathrm{PbMoO}_{4}$ on the level of hundreds $\mathrm{Bq} / \mathrm{kg}$. The ${ }^{210} \mathrm{~Pb}$ activity can be reduced by several orders of magnitude by production of lead containing scintillators from archaeological lead [89, 61, 40]. Despite being a very weak, the $2 \beta^{-}$activity of ${ }^{48} \mathrm{Ca},{ }^{100} \mathrm{Mo},{ }^{116} \mathrm{Cd}, \alpha$ activity of ${ }^{180} \mathrm{~W}$ can also be sources of background in low counting experiments. For instance, presence of $2 \beta^{-}$active ${ }^{48} \mathrm{Ca}$ in $\mathrm{CaMoO}_{4}$ makes problematic application of the crystal scintillator in high sensitivity experiments to search for $0 \nu 2 \beta^{-}$decay of ${ }^{100} \mathrm{Mo}$, while the $2 \nu 2 \beta^{-}$decays of ${ }^{100} \mathrm{Mo}$ will generate unavoidable background in the ROI of ${ }^{116} \mathrm{Cd}$ in a detector based on $\mathrm{CdMoO}_{4}$ crystal scintillators [90].

\subsection{Isotopically enriched scintillators}

High concentration of isotope of interest is an important requirement to scintillation materials to be used in $2 \beta$ decay experiments that suggests development of scintillators from enriched isotopes. The request for radiopure crystal scintillators from isotopically enriched materials imposes specific requirements to the production process: maximal yield of the ready to use scintillation elements and minimal losses of the enriched material. Typical purity grade of isotopically enriched materials (both chemical and radioactive contaminations) is much worse than that required for radiopure crystal growth. Therefore, a production cycle of enriched scintillators should include development of specific purification, crystal growth and treatment protocols, recovery of costly enriched materials from all the scraps [91.

First crystal scintillators from enriched materials were developed by der Mateosian and Goldhaber [92]. Europium-activated calcium fluoride crystal scintillators enriched in ${ }^{48} \mathrm{Ca}$ $\left({ }^{48} \mathrm{CaF}_{2}(\mathrm{Eu})\right)$ and depleted in ${ }^{48} \mathrm{Ca}\left({ }^{48 d e p l} \mathrm{CaF}_{2}(\mathrm{Eu})\right)$ were grown to search for the $2 \beta^{-}$decay of ${ }^{48} \mathrm{Ca}$ at the level of sensitivity $\lim T_{1 / 2}^{0 \nu 2 \beta^{-}}=2 \times 10^{20}$ yr [92]. However, the crystals enriched in ${ }^{48} \mathrm{Ca}$ were substantially contaminated by $\mathrm{U} / \mathrm{Th}$.

Radiopure cadmium tungstate crystal scintillator enriched in ${ }^{116} \mathrm{Cd}\left({ }^{116} \mathrm{CdWO}_{4}\right)$ was developed for the Solotvina experiment [93] by using the convenient Czochralski growth process. In the experiment at the Solotvina Underground laboratory the two neutrino mode of the $2 \beta^{-}$decay of ${ }^{116} \mathrm{Cd}$ was observed. For the neutrinoless $2 \beta^{-}$decay of ${ }^{116} \mathrm{Cd}$ the half-life limit $T_{1 / 2} \geq 1.7 \times 10^{23}$ yr was set at $90 \%$ C.L., which corresponds to the effective Majorana neutrino mass limit $\left\langle m_{\nu}\right\rangle \leq 1.7 \mathrm{eV}$ [81].

Cadmium tungstate crystal scintillator from enriched to $66 \%$ isotope cadmium $106\left({ }^{106} \mathrm{CdWO}_{4}\right)$ with mass $231 \mathrm{~g}$ was developed for the double beta experiments with ${ }^{106} \mathrm{Cd}$, that is one of the most promising double beta plus decay nuclei. The deep purification of initial materials [94, 95], particularly application of vacuum distillation with filtering on getter filters to purify the enriched cadmium samples, together with the low-thermal-gradient Czochralski method to grow the crystal [96, 97, resulted in unprecedented high optical quality of the crystal scintillator [98. The ${ }^{106} \mathrm{CdWO}_{4}$ crystal is now used in experiments to search for $2 \beta$ processes in ${ }^{106} \mathrm{Cd}$, with preliminary results reported in [41, 40]. In the last configuration of the experiment, the ${ }^{106} \mathrm{CdWO}_{4}$ scintillator is viewed by a low-background $\mathrm{PMT}$ through a $\mathrm{PbWO}_{4}$ light-guide produced from deeply purified archaeological lead. The new stage of experiment is now running in the DAMA/Crys low-background set-up at the Gran Sasso underground laboratory (Italy). The ${ }^{106} \mathrm{CdWO}_{4}$ detector is operated in coincidence with two large volume $\mathrm{CdWO}_{4}$ crystal scintillators in close geometry aiming at increase of the detection efficiency to the $2 \beta$ processes in 
${ }^{106} \mathrm{Cd}$ with emission of $\gamma$ quanta [99]. The sensitivity of the experiment, e.g., to two neutrino mode of $\varepsilon \beta^{+}$decay of ${ }^{106} \mathrm{Cd}$ is on the level of the theoretical estimations of the process half-life $T_{1 / 2} \sim 10^{22} \mathrm{yr}$.

A large volume cadmium tungstate crystal (mass of the crystal boule $1868 \mathrm{~g}$ ) from isotopically enriched cadmium $116\left({ }^{116} \mathrm{CdWO}_{4}\right)$ was developed also by the low-thermal-gradient Czochralski technique [82. The boule was cut into several parts, two of them with a total mass $1162 \mathrm{~g}$ were used in the Aurora experiment at the Gran Sasso underground laboratory. After more than 25 thousand hours of data taking the half-life of ${ }^{116} \mathrm{Cd}$ relatively to the two neutrino $2 \beta^{-}$decay of ${ }^{116} \mathrm{Cd}$ is measured with the highest up-to-date accuracy as $T_{1 / 2}=[2.69 \pm 0.02$ (stat.) \pm 0.14 (syst.) $] \times 10^{19} \mathrm{yr}$. The systematic error of the $T_{1 / 2}$ value is mainly due to the radioactive contamination of the crystal by ${ }^{238} \mathrm{U}$ at the level of $\sim 0.6 \mathrm{mBq} / \mathrm{kg}$. A new improved limit on the $0 \nu 2 \beta^{-}$decay of ${ }^{116} \mathrm{Cd}$ has been set as $T_{1 / 2} \geq 2.4 \times 10^{23}$ yr at $90 \%$ C.L., that corresponds to the limit on the effective Majorana neutrino mass $\left\langle m_{\nu}\right\rangle \leq(1.2-1.5)$ $\mathrm{eV}$ (depending on the nuclear matrix elements used in the estimations of the neutrino mass limit). New improved limits on other double beta decay processes in ${ }^{116} \mathrm{Cd}$ (decays with majoron emission, transitions to excited levels) were set at the level of $T_{1 / 2}>10^{21}-10^{22} \mathrm{yr}$ [100, 42]. The experiment up to date is the most sensitive study of the ${ }^{116} \mathrm{Cd}$ double beta decay.

An important advantage of the low-thermal-gradient Czochralski method is very high yield of crystalline boules ( $87 \%$ of the initial ${ }^{106} \mathrm{CdWO}_{4}$ and ${ }^{116} \mathrm{CdWO}_{4}$ powders). Besides, a much lower overheating of the melt (in comparison to the conventional Czochralski process) allowed to achieve very low evaporation losses of costly enriched cadmium in the growing process. The total irrecoverable losses of enriched cadmium in the ${ }^{106} \mathrm{CdWO}_{4}$ and ${ }^{116} \mathrm{CdWO}_{4}$ developments does not exceed $2 \%$ (with the main losses appeared due to the purification of the cadmium samples by vacuum distillation, that can be further reduced by development of especial equipment to minimize the losses).

Calcium molybdate crystal scintillators enriched in molybdenum 100, from calcium depleted in calcium 48 were developed for the AMoRE $2 \beta$ experiment [39, 101] aiming at search for $0 \nu 2 \beta^{-}$ decay of ${ }^{100} \mathrm{Mo}$ by using cryogenic scintillating bolometers. Using of the depleted calcium is requested to avoid contribution of the $2 \nu 2 \beta^{-}$events of ${ }^{48} \mathrm{Ca}\left(Q_{2 \beta}=4268 \mathrm{keV}\right)$ to the ROI of the AMoRE experiment (energy of the expected $0 \nu 2 \beta^{-}$peak of ${ }^{100} \mathrm{Mo}$ is $Q_{2 \beta}=3034 \mathrm{keV}$ ). The crystal scintillators were produced by the conventional Czochralski method.

Zinc $\left(\mathrm{ZnMoO}_{4}\right)$ and lithium $\left(\mathrm{Li}_{2} \mathrm{MoO}_{4}\right)$ molybdate crystal scintillators were extensively studied by the LUMINEU $2 \beta$ project to develop high sensitivity detectors to search for $0 \nu 2 \beta^{-}$ decay of ${ }^{100}$ Mo. High quality crystal scintillators were obtained after the R\&D that included deep purification of molybdenum and application of the low-thermal-gradient Czochralski process to grow crystals [67, 102, 103, 104, 63]. Both the scintillation materials were then produced from enriched ${ }^{100} \mathrm{Mo}$ [105, 64]. Similar to the enriched cadmium tungstate crystals, rather large crystal boules yield $\left(\approx 84 \%\right.$ and $\approx 84 \%$ of the initial charge for $\mathrm{Zn}^{100} \mathrm{MoO}_{4}$ and $\mathrm{Li}_{2}^{100} \mathrm{MoO}_{4}$, respectively) and low losses of molybdenum $\left(\approx 4 \%\right.$ and $\approx 3 \%$ ) were achieved in the $\mathrm{Zn}^{100} \mathrm{MoO}_{4}$ and $\mathrm{Li}_{2}^{100} \mathrm{MoO}_{4}$ crystals production.

The radiopurity level of the developed enriched cadmium tungstate, lithium, calcium and zinc molybdate crystal scintillators is presented in Table 4. 


\section{Prospects for the next generation $0 \nu 2 \beta^{-}$experiments with crystal scintillators}

Two big projects aiming at construction of large scale experiments to search for $0 \nu 2 \beta^{-}$decay on the level of inverted hierarchy of the neutrino masses intend to use the technique of low temperature scintillating bolometers.

The AMoRE (Advanced Mo-based Rare process Experiment) project [106, 107] utilizes Metal Magnetic Calorimeter sensors to measure the heat and scintillation signals from calcium molybdate crystal scintillators [108]. Now the experiment is running in a pilot phase with $\approx 1.5 \mathrm{~kg}$ of enriched in ${ }^{100} \mathrm{Mo}$ and depleted in ${ }^{48} \mathrm{Ca}{ }^{48 d e p l} \mathrm{Ca}^{100} \mathrm{MoO}_{4}$ crystal scintillators at the YangYang underground laboratory (Korea). The next phase of the experiment, AMoRE I, should start in the beginning of 2018 and utilize the same low background cryostat with $\approx 5$

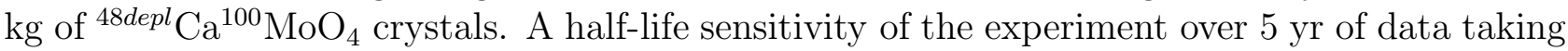
is expected to be at the level of $T_{1 / 2} \sim 10^{25}$ years at $90 \%$ C.L. assuming zero background. A next stage AMoRE II experiment with $\approx 200 \mathrm{~kg}$ molybdate crystal scintillators is planned in a deeper underground site in Korea that is under construction now. The goal of AMoRE II is to explore the inverted hierarchy of neutrino mass with a half-life sensitivity at the level of $T_{1 / 2} \sim 5 \times 10^{26}$ years that corresponds to the effective neutrino mass $\left\langle m_{\nu}\right\rangle \sim 0.02-0.03$ $\mathrm{eV}$ (depending on the nuclear matrix elements calculations). R\&D of several molybdenum containing crystal scintillators is in progress to replace $\mathrm{CaMoO}_{4}$ in the $\mathrm{AMoRE}$ II phase.

The aim of CUPID (Cuore Upgrade with Particle IDentification) is to construct a tonnescale low temperature experiment to probe the Majorana nature of neutrinos and discover the lepton number violation in the inverted hierarchy region of the neutrino mass [109, 110]. The experiment is intended to exploit the current CUORE infrastructure [11] as much as possible. The isotopes ${ }^{82} \mathrm{Se},{ }^{100} \mathrm{Mo},{ }^{116} \mathrm{Cd}$ and ${ }^{130} \mathrm{Te}$, embedded in $\mathrm{ZnSe}, \mathrm{ZnMoO}_{4}$ or $\mathrm{Li}_{2} \mathrm{MoO}_{4}, \mathrm{CdWO}_{4}$ and $\mathrm{TeO}_{2}$ crystals, are considered as the candidate nuclei. A substantial reduction of background to the level of $<0.02$ counts $/($ ton $\times y r)$ in the ROI can be achieved thanks to particle identification by simultaneous registration of thermal and scintillation signals (Cherenkov radiation in the case of $\left.\mathrm{TeO}_{2}\right)$. With an energy resolution at endpoint (FWHM $=5 \mathrm{keV}$ ) CUPID could reach a $90 \%$ C.L. sensitivity of $\lim T_{1 / 2} \sim(2-5) \times 10^{27}$ yr after 10 years of operation, that corresponds to a range of the effective Majorana neutrino masse sensitivity $\lim \left\langle m_{\nu}\right\rangle \sim 6-19$ $\mathrm{meV}$, depending on the estimate of the nuclear matrix elements.

The CUPID R\&D program is in progress aiming at development of purification and crystallization procedures, new detector technologies, development of existing and search for new scintillation materials containing the candidate elements. A first array of enriched $\mathrm{Zn}^{82} \mathrm{Se}$ bolometers is running at the Gran Sasso underground laboratory [112. A comprehensive R\&D was performed in the framework of the LUMINEU project to develop high performance $\mathrm{ZnMoO}_{4}$ and $\mathrm{Li}_{2} \mathrm{MoO}_{4}$ cryogenic detectors for $0 \nu 2 \beta^{-}$experiment with ${ }^{100} \mathrm{Mo}$. Large volume (production of scintillation elements $5 \mathrm{~cm}$ in diameter and $5 \mathrm{~cm}$ height was well established), highly radiopure (activities of ${ }^{226} \mathrm{Ra}$ and ${ }^{228} \mathrm{Th}$ less than $5 \mu \mathrm{Bq} / \mathrm{kg}$ ), high optical quality crystal scintillators (including two large volume samples from enriched ${ }^{100} \mathrm{Mo}$ ) were developed [64]. Nevertheless, presence of second crystal phase inclusions remains the main problem both in production and bolometric performance of $\mathrm{ZnMoO}_{4}$ crystals. In parallel to the $\mathrm{ZnMoO}_{4}$ development, an $\mathrm{R} \& \mathrm{D}$

of lithium molybdate was pursued. Several underground tests of $\mathrm{Li}_{2} \mathrm{MoO}_{4}$ and $\mathrm{Li}_{2}^{100} \mathrm{MoO}_{4}$ detector modules, as well as features of the production process, encouraged choice of the ma- 
terial for the construction of the CUPID-0/Mo demonstrator. The production of 20 enriched $\mathrm{Li}_{2}^{100} \mathrm{MoO}_{4}$ scintillation elements $4.4 \mathrm{~cm}$ in diameter and $4.5 \mathrm{~cm}$ height for the first stage of the experiment was completed recently [113, 114].

A first test of $34.5 \mathrm{~g}$ enriched ${ }^{116} \mathrm{CdWO}_{4}$ sample at milli-Kelvin temperature confirmed a high performance of the material in terms of energy resolution and particle discrimination capability 115. The aim of the CYGNUS project (CrYoGenic search for NeUtrinoleSs double beta decay of cadmium) is investigation of $2 \beta^{-}$decay of ${ }^{116} \mathrm{Cd}$ with $1.16 \mathrm{~kg}$ of enriched radiopure ${ }^{116} \mathrm{CdWO}_{4}$ crystal scintillators [82] as cryogenic scintillating bolometers. The proposed experiment will take advantage of the EDELWEISS ultra-low background set-up located at the deepest European underground Laboratory in Modane (France). The project should further prove suitability of the material for the CUPID project.

Developments of $\mathrm{TeO}_{2}$ detectors with Cherenkov emission readout 3 are carried out by using different approaches to detect very weak Cherenkov light from $\mathrm{TeO}_{2}$ crystals [117, 118, 120, 119, 121.

\section{Conclusions}

Crystal scintillators are successfully used in $2 \beta$ experiments thanks to the possibility to realize calorimetric "source = detector" approach for several nuclei with a high detection efficiency. A very low radiopurity level of tungstate and molybdate crystals (including the scintillators from isotopically enriched materials) is achieved thanks to the deep purification of the initial materials, application of the low-thermal-gradient Czochralski process to grow the crystals, and 2nd crystallization. A high energy resolution (a few $\mathrm{keV}$ ) and excellent particle discrimination ability of the low temperature scintillating bolometers make the detectors promising for the next generation $0 \nu 2 \beta^{-}$experiments aiming at test the inverted neutrino mass scheme and even go toward the normal neutrino mass hierarchy.

\section{Acknowledgments}

Author gratefully acknowledges support from the "Jean d'Alembert" Grants program (Project CYGNUS) of the University of Paris-Saclay.

\footnotetext{
${ }^{3}$ The idea to use Cherenkov signal for $\beta$ and $\alpha$ events discrimination was first proposed by Tabarelli de Fatis in 2010 [116]).
} 


\section{References}

[1] V.I. Tretyak, Yu.G. Zdesenko, Tables of double beta decay - an update, At. Data Nucl. Data Tables 80, 83 (2002).

[2] R. Saakyan, Two-Neutrino Double-Beta Decay, Annu. Rev. Nucl. Part. Sci. 63, 503 (2013).

[3] A.S. Barabash, Average and recommended half-life values for two-neutrinodouble beta decay, Nucl. Phys. A 935, 52 (2015).

[4] J. Schechter, J.W.F. Valle, Neutrinoless double- $\beta$ decay in $\mathrm{SU}(2) \times \mathrm{U}(1)$ theories, Phys. Rev. D 25, 2951 (1982).

[5] J. Barea, J. Kotila, F. Iachello, Limits on Neutrino Masses from Neutrinoless Double- $\beta$ Decay, Phys. Rev. Lett. 109, 042501 (2012).

[6] W. Rodejohann, Neutrino-less double beta decay and particle physics, J Phys. G 39, 124008 (2012).

[7] F.F. Deppisch, M. Hirsch, H. Päs, Neutrinoless double-beta decay and physics beyond the standard model, J Phys. G 39, 124007 (2012).

[8] S.M. Bilenky, C. Giunti, Neutrinoless double-beta decay: A probe of physics beyond the Standard Model, Int. J Mod. Phys. A 30, 1530001 (2015).

[9] S. Dell'Oro, S. Marcocci, M. Viel, F. Vissani, Neutrinoless Double Beta Decay: 2015 Review, AHEP, 2016, 2162659 (2016).

[10] J.D. Vergados, H. Ejiri, F. Šimkovic, Neutrinoless double beta decay and neutrino mass, Int. J. Mod. Phys. E 25, 1630007 (2016).

[11] S.R. Elliott, Recent progress in double beta decay, Mod. Phys. Lett. A 27, 123009 (2012).

[12] A. Giuliani, A. Poves, Neutrinoless Double-Beta Decay, AHEP 2012, 857016 (2012).

[13] O. Cremonesi, M. Pavan, Challenges in Double Beta Decay, AHEP 2014, 951432 (2014).

[14] J.J. Gómez-Cadenas, J. Martín-Albo, Phenomenology of Neutrinoless Double Beta Decay, in Proc. Gran Sasso Summer Institute 2014 Hands-On Experimental Underground Physics at LNGS (GSSI14), INFN - Laboratori Nazionali del Gran Sasso, Assergi, Italy, 22 September - 03 October 2014, PoS(GSSI14)004.

[15] X. Sarazin, Review of Double Beta Experiments, J Phys.: Conf. Ser. 593, 012006 (2015).

[16] M. Agostini et al., Background-free search for neutrinoless double- $\beta$ decay of ${ }^{76}$ Ge with GERDA, Nature 544, 47 (2017).

[17] J.B. Albert et al. (The EXO-200 Collaboration), Search for Majorana neutrinos with the first two years of EXO-200 data, Nature 510, 229 (2014). 
[18] K. Alfonso et al. (CUORE Collaboration), Search for Neutrinoless Double-Beta Decay of ${ }^{130}$ Te with CUORE-0, Phys. Rev. Lett. 115, 102502 (2015).

[19] R. Arnold et al., Results of the search for neutrinoless double- $\beta$ decay in ${ }^{100}$ Mo with the NEMO-3 experiment, Phys. Rev. D 92, 072011 (2015).

[20] A. Gando et al. (KamLAND-Zen Collaboration), Search for Majorana Neutrinos Near the Inverted Mass Hierarchy Region with KamLAND-Zen, Phys. Rev. Lett. 117, 082503 (2017).

[21] J. Engel, J. Menéndez, Status and future of nuclear matrix elements for neutrinoless double-beta decay: a review, Rep. Prog. Phys. 80, 046301 (2017).

[22] J. Maalampi, J. Suhonen, Neutrinoless Double $\beta^{+} /$EC Decays, AHEP 2013, 505874 (2013).

[23] M. Hirsch, K. Muto, T. Oda, H.V. Klapdor-Kleingrothaus, Nuclear structure calculation of $\beta^{+} \beta^{+}, \beta^{+} / \mathrm{EC}$ and EC/EC decay matrix elements, Z. Phys. A 347, 151 (1994).

[24] R.G. Winter, Double K capture and single K capture with positron emission, Phys. Rev. 100, 142 (1955).

[25] M.B. Voloshin, G.V. Mitselmakher, R.A. Eramzhyan, Conversion of an atomic electron into a positron and double decay, JETP Lett. 35, 656 (1982).

[26] J. Bernabeu, A. De Rujula, C. Jarlskog, Neutrinoless double electron capture as a tool to measure the electron neutrino mass, Nucl. Phys. B 223, 15 (1983).

[27] Z. Sujkowski, S. Wycech, Neutrinoless double electron capture: A tool to search for Majorana neutrinos, Phys. Rev. C 70, 052501 (2004).

[28] M.I. Krivoruchenko , F. Šimkovic, D. Frekers, A. Faessler, Resonance enhancement of neutrinoless double electron capture, Nucl. Phys. A 859, 140 (2011).

[29] J. Kotila, J. Barea, F. Iachello, Neutrinoless double-electron capture, Phys. Rev. C 89, 064319 (2014).

[30] K. Blaum et al., Neutrinoless double electron capture, review in preparation.

[31] S.M. Bilenky, J.A. Grifols, The possible test of the calculations of nuclear matrix elements of the $(\beta \beta)_{0 \nu}$-decay, Phys. Lett. B 550, 154 (2002).

[32] F.A. Danevich et al., $\mathrm{CdWO}_{4}, \mathrm{ZnSe}$ and $\mathrm{ZnWO}_{4}$ scintillators in studies of $2 \beta$-processes, Instr. Exp. R. 32, 1059 (1989).

[33] M. Minova, K. Itakura, S. Moriyama, W. Ootani, Measurement of the property of cooled lead molybdte as a scintillator, Nucl. Instr. Meth. A 320. 500 (1992).

[34] G. Angloher et al., New limits on double electron capture of ${ }^{40} \mathrm{Ca}$ and ${ }^{180} \mathrm{~W}, J$ Phys. G: Nucl. Part. Phys. 43, 095202 (2016). 
[35] S. Umehara et al., Neutrino-less double- $\beta$ decay of ${ }^{48} \mathrm{Ca}$ studied by $\mathrm{CaF}_{2}(\mathrm{Eu})$ scintillators, Phys. Rev. C 78, 058501 (2008).

[36] P. Belli et al., Final results of an experiment to search for $2 \beta$ processes in zinc and tungsten with the help of radiopure $\mathrm{ZnWO}_{4}$ crystal scintillators, J Phys. G: Nucl. Part. Phys. 38, 115107 (2011).

[37] L. Cardani et al., First bolometric measurement of the two neutrino double beta decay of ${ }^{100} \mathrm{Mo}$ with a $\mathrm{ZnMoO}_{4}$ crystals array, J Phys. G 41, 075204 (2014).

[38] D.V. Poda et al., ${ }^{100}$ Mo-enriched $\mathrm{Li}_{2} \mathrm{MoO}_{4}$ scintillating bolometers for $0 \nu 2 \beta$ decay search: from LUMINEU to CUPID-0/Mo projects , to appear in Proceedings of MEDEX'17 meeting (Prague, May 29 - June 2, 2017).

[39] J.H. So et al., Scintillation properties and internal background study of ${ }^{40} \mathrm{Ca}^{100} \mathrm{MoO}_{4}$ crystal scintillators for neutrino-less double beta decay search, IEEE Trans. Nucl. Sci. 59, 2214 (2012).

[40] P. Belli et al., Search for $2 \beta$ decay of ${ }^{106} \mathrm{Cd}$ with an enriched ${ }^{106} \mathrm{CdWO}_{4}$ crystal scintillator in coincidence with four HPGe detectors, Phys. Rev. C 93, 045502 (2016).

[41] P. Belli et al., Search for double- $\beta$ decay processes in ${ }^{106} \mathrm{Cd}$ with the help of a ${ }^{106} \mathrm{CdWO}_{4}$ crystal scintillator, Phys. Rev. C 85, 044610 (2012).

[42] O.G. Polischuk et al., Investigation of $2 \beta$ decay of ${ }^{116} \mathrm{Cd}$ with the help of enriched ${ }^{116} \mathrm{CdWO}_{4}$ crystal scintillators, to appear in Proceedings of MEDEX'17 meeting (Prague, May 29 - June 2, 2017).

[43] R. Cerulli et al., Performances of a $\mathrm{BaF}_{2}$ detector and its application to the search for $\beta \beta$ decay modes in ${ }^{130} \mathrm{Ba}$, Nucl. Instr. Meth. A 525, 535 (2004).

[44] P. Belli et al., Search for $2 \beta$ decay of cerium isotopes with $\mathrm{CeCl}_{3}$ scintillator, $J$ Phys. G: Nucl. Part. Phys. 38, 015103 (2011).

[45] F.A. Danevich et al., Quest for double beta decay of ${ }^{160} \mathrm{Gd}$ and Ce isotopes, Nucl. Phys. A 694, 375 (2001).

[46] M. Wang et al., The Ame2016 atomic mass evaluation, Chin. Phys. C 41, 030003 (2017).

[47] J. Meija et al., Isotopic compositions of the elements 2013 (IUPAC Technical Report), Pure Appl. Chem. 88, 293 (2016).

[48] Yu.G. Zdesenko, The future of double $\beta$ decay research, Rev. Mod. Phys. 74, 663 (2002).

[49] G.J. Feldman, R.D. Cousins, Unified approach to the classical statistical analysis of small signals, Phys. Rev. D 57, 3873 (1998).

[50] F.A. Danevich et al., $\mathrm{MgWO}_{4}-\mathrm{A}$ new crystal scintillator, Nucl. Instr. Meth. A 608, 107 (2009).

[51] Yu.G. Zdesenko et al., Scintillation properties and radioactive contamination of $\mathrm{CaWO}_{4}$ crystal scintillators, Nucl. Instr. Meth. A 538, 657 (2005). 
[52] C. Cozzini et al,. Detection of the natural $\alpha$ decay of tungsten, Phys. Rev. C 70, 064606 (2004).

[53] F.A. Danevich et al. Impact of geometry on light collection efficiency of scintillation detectors for cryogenic rare event searches, Nucl. Instr. Meth. B 336, 26 (2014).

[54] L.L. Nagornaya et al., Growth of $\mathrm{ZnWO}_{4}$ Crystal Scintillators for High Sensitivity $2 \beta$ Experiments, IEEE Trans. Nucl. Sci. 55, 1469 (2008).

[55] N. Casali et al., Cryogenic Detectors for Rare Alpha Decay Search: A New Approach, J Low Temp. Phys. 184, 952 (2016).

[56] F.A. Danevich et al., $\mathrm{ZnWO}_{4}$ crystals as detectors for $2 \beta$ decay and dark matter experiments, Nucl. Instr. Meth. A 544, 553 (2005).

[57] L. Bardelli et al., Further study of $\mathrm{CdWO}_{4}$ crystal scintillators as detectors for high sensitivity $2 \beta$ experiments: Scintillation properties and pulse-shape discrimination, Nucl. Instr. Meth. A 569, 743 (2006).

[58] C. Arnaboldi et al., $\mathrm{CdWO}_{4}$ scintillating bolometer for Double Beta Decay: Light and heat anticorrelation, light yield and quenching factors, Astropart. Phys. 34, 143 (2010).

[59] D.R. Artusa et al., Exploring the neutrinoless double beta decay in the inverted neutrino hierarchy with bolometric detectors, Eur. Phys. J C 74, 3096 (2014).

[60] L. Bardelli et al., Pulse-shape discrimination with $\mathrm{PbWO}_{4}$ crystal scintillators, Nucl. Instr. Meth. A 584, 129 (2008).

[61] J.W. Beeman et al., New experimental limits on the $\alpha$ decays of lead isotopes, Eur. Phys. $J$ A 49, 50 (2013).

[62] F.A. Danevich et al., Application of $\mathrm{PbWO}_{4}$ crystal scintillators in experiment to search for $2 \beta$ decay of ${ }^{116} \mathrm{Cd}$, Nucl. Instr. Meth. A 556, 259 (2006).

[63] T.B. Bekker et al., Aboveground test of an advanced $\mathrm{Li}_{2} \mathrm{MoO}_{4}$ scintillating bolometer to search for neutrinoless double beta decay of ${ }^{100} \mathrm{Mo}$, Astropart. Phys. 72, 38 (2016).

[64] E. Armengaud et al., Development of ${ }^{100}$ Mo-containing scintillating bolometers for a highsensitivity neutrinoless double-beta decay search, arXiv:1704.01758v1 [physics.ins-det], to appear in Eur. Phys. J C.

[65] A.N. Annenkov et al., Development of $\mathrm{CaMoO}_{4}$ crystal scintillators for a double beta decay experiment with ${ }^{100} \mathrm{Mo}$, Nucl. Instr. Meth. A 584, 334 (2008).

[66] G.B. Kim et al., A $\mathrm{CaMoO}_{4}$ Crystal Low Temperature Detector for the AMoRE Neutrinoless Double Beta Decay Search, AHEP 2015, 817530 (2015).

[67] D.M. Chernyak et al., Optical, luminescence and thermal properties of radiopure $\mathrm{ZnMoO}_{4}$ crystals used in scintillating bolometers for double beta decay search, Nucl. Instr. Meth. A 729, 856 (2013). 
[68] J.W. Beeman et al., A next-generation neutrinoless double beta decay experiment based on $\mathrm{ZnMoO}_{4}$ scintillating bolometers, Phys. Lett. B 710, 318 (2012).

[69] H. Jiang et al., Growth and Scintillation Characterizations of $\mathrm{SrMoO}_{4}$ Single Crystals, $J$ Korean Phys. Soc. 63, 2018 (2013).

[70] F.A. Danevich et al., Feasibility study of $\mathrm{PbWO}_{4}$ and $\mathrm{PbMoO}_{4}$ crystal scintillators for cryogenic rare events experiments, Nucl. Instr. Meth. A 622, 608 (2010).

[71] T. Fazzini et al., Pulse-shape discrimination with $\mathrm{CdWO}_{4}$ crystal scintillators, Nucl. Instr. Meth. A 410, 213 (1998).

[72] C. Arnaboldi et al., Characterization of ZnSe scintillating bolometers for Double Beta Decay, Astropart. Phys. 34, 344 (2011).

[73] V.I. Tretyak, Semi-empirical calculation of quenching factors for ions in scintillators, Astropart. Phys. 33, 40 (2010).

[74] L. Gironi, Scintillating bolometers for Double Beta Decay search, Nucl. Instr. Meth. A 617, 478 (2010).

[75] S. Cebrián, et al., Bolometric WIMP search at Canfranc with different absorbers, Astropart. Phys. 21, 23 (2004).

[76] P. Belli et al., Radioactive contamination of $\mathrm{ZnWO}_{4}$ crystal scintillators, Nucl. Instr. Meth. A 626-627, 31 (2011).

[77] F.A. Danevich et al., Investigation of $\beta^{+} \beta^{+}$and $\beta^{+} / \mathrm{EC}$ decay of ${ }^{106} \mathrm{Cd}$, Z. Phys. A 355, 433 (1996).

[78] P. Belli et al., Investigation of $\beta$ decay of ${ }^{113} \mathrm{Cd}$, Phys. Rev. C 76, 064603 (2007).

[79] A.Sh. Georgadze et al., Evaluation of activities of impurity radionuclides in cadmium tungstate crystals, Instr. Exp. Technique 39, 191 (1996).

[80] F.A. Danevich et al., $\alpha$ activity of natural tungsten isotopes, Phys. Rev. C 67, 014310 (2003).

[81] F.A. Danevich et al., Search for $2 \beta$ decay of cadmium and tungsten isotopes: Final results of the Solotvina experiment, Phys. Rev. C 68, 035501 (2003).

[82] A.S. Barabash et al., Low background detector with enriched ${ }^{116} \mathrm{CdWO}_{4}$ crystal scintillators to search for double $\beta$ decay of ${ }^{116} \mathrm{Cd}$, JINST 6, P08011 (2011).

[83] D.V. Poda et al., $\mathrm{CdWO}_{4}$ crystal scintillators from enriched isotopes for double beta decay experiments, Rad. Meas. 56, 66 (2013).

[84] A.S. Barabash et al., Improvement of radiopurity level of enriched ${ }^{116} \mathrm{CdWO}_{4}$ and $\mathrm{ZnWO}_{4}$ crystal scintillators by recrystallization, Nucl. Instr. Meth. A 833, 77 (2016).

[85] A. Luqman et al., Simulations of background sources in AMoRE-I experiment, Nucl. Instr. Meth. A 855, 140 (2017). 
[86] H.S. Lee et al., Development of low-background CsI(Tl) crystals for WIMP search, Nucl. Instr. Meth. A 571, 644 (2007).

[87] C. Arnaboldi et al., Production of high purity $\mathrm{TeO}_{2}$ single crystals for the study of neutrinoless double beta decay, J Cryst. Growth 312, 2999 (2010).

[88] F.A. Danevich et al., Effect of recrystallisation on the radioactive contamination of $\mathrm{CaWO}_{4}$ crystal scintillators, Nucl. Instr. Meth. A 631, 44 (2011).

[89] A. Alessandrello et al., Measurements of internal radioactive contamination in samples of Roman lead to be used in experiments on rare events, Nucl. Instr. Meth. A 409, 451 (1998).

[90] M.X. Xue et al., Study of $\mathrm{CdMoO}_{4}$ crystal for a neutrinoless double beta decay experiment with ${ }^{116} \mathrm{Cd}$ and ${ }^{100}$ Mo nuclides, Chin. Phys. C 41, 046002 (2017).

[91] F.A. Danevich, Development of Crystal Scintillators From Enriched Isotopes for Double Decay Experiments, IEEE Trans. Nucl. Sci. 59, 2207 (2012).

[92] E. der Mateosian, M. Goldhaber, Limits for Lepton-Nonconserving Double Bea Decay of $\mathrm{Ca}^{48}$, Phys. Rev. 146, 810 (1966).

[93] F.A. Danevich et al., Search for $2 \beta$ decay of ${ }^{116} \mathrm{Cd}$ with the help of a ${ }^{116} \mathrm{CdWO}_{4}$ scintillator, JETP Lett. 49, 476 (1989).

[94] R. Bernabey et al., Production of high-pure $\mathrm{Cd}$ and ${ }^{106} \mathrm{Cd}$ for $\mathrm{CdWO}_{4}$ and ${ }^{106} \mathrm{CdWO}_{4}$ scintillators, Metallofizika i Noveishie Tekhnologii 30 (spec. issue), 477 (2008) (in Russian).

[95] G.P. Kovtun et al., Production of radiopure natural and isotopically enriched cadmium and zinc for low background scintillators, Functional Materials 18, 121 (2011).

[96] A.A. Pavlyuk et al., Low Thermal Gradient technique and method for large oxide crystals growth from melt and flux, in Proceeding of the APSAM-92, Shanghai, 26-29 April 1992, Asia Pacific Society for Advanced Materials, Institute of Materials Research (Tohoku University, Sendai, Japan, 1993), p. 164.

[97] E.N. Galashov et al., The growth of $\mathrm{ZnWO}_{4}$ and $\mathrm{CdWO}_{4}$ single crystals from melt by the low thermal gradient Czochralski technique, Crystallogr. Rep. 54, 689 (2009).

[98] P. Belli et al., Development of enriched ${ }^{106} \mathrm{CdWO}_{4}$ crystal scintillators to search for double $\beta$ decay processes in ${ }^{106} \mathrm{Cd}$, Nucl. Instr. Meth. A 615, 301 (2010).

[99] R. Cerulli et al., Search for double beta decay in ${ }^{106} \mathrm{Cd}$ in the DAMA/Crys setup, to appear in Proceedings of MEDEX'17 meeting (Prague, May 29 - June 2, 2017).

[100] F.A. Danevich et al., Search for double beta decay of ${ }^{116} \mathrm{Cd}$ with enriched ${ }^{116} \mathrm{CdWO}_{4}$ crystal scintillators (Aurora experiment), J Phys. Conf. Ser. 718, 062009 (2016).

[101] J.H. So et al., A study of $\mathrm{CaMoO}_{4}$ crystals for the AMoRE experiment, IEEE Nucl. Sci. Symp., N41-8, 1987 (2012). 
[102] L. Berge et al., Purification of molybdenum, growth and characterization of medium volume $\mathrm{ZnMoO}_{4}$ crystals for the LUMINEU program, JINST 9, P06004 (2014).

[103] E. Armengaud et al., Development and underground test of radiopure $\mathrm{ZnMoO}_{4}$ scintillating bolometers for the LUMINEU 0 $2 \beta$ project, JINST 10, P05007 (2015).

[104] D.M. Chernyak et al., Effect of tungsten doping on $\mathrm{ZnMoO}_{4}$ scintillating bolometer performance, Opt. Mat. 49, 67 (2015).

[105] A.S. Barabash et al., Enriched $\mathrm{Zn}^{100} \mathrm{MoO}_{4}$ scintillating bolometers to search for $0 \nu 2 \beta$ decay of ${ }^{100}$ Mo with the LUMINEU experiment, Eur. Phys. J C 74, 3133 (2014).

[106] H. Bhang et al., AMoRE experiment: a search for neutrinoless double beta decay of ${ }^{100} \mathrm{Mo}$ isotope with ${ }^{40} \mathrm{Ca}^{100} \mathrm{MoO}_{4}$ cryogenic scintillation detector, J Phys. Conf. Ser. 375, 042023 (2012).

[107] V. Alenkov et al., Technical Design Report for the AMoRE $0 \nu \beta \beta$ Decay Search Experiment, arXiv:1512.05957v1 [physics.ins-det].

[108] S.J.Lee et al., The development of a cryogenic detector with $\mathrm{CaMoO}_{4}$ crystals for neutrinoless double beta decay search, Astropart. Phys. 34, 732 (2011).

[109] G. Wang et al., CUPID: CUORE (Cryogenic Underground Observatory for Rare Events) Upgrade with Particle IDentification, arXiv:1504.03599v1 [physics.ins-det].

[110] G. Wang et al., R\&D towards CUPID ( CUORE Upgrade with Particle IDentification), arXiv:1504.03612v1 [physics.ins-det].

[111] D.R. Artusa et al., Searching for Neutrinoless Double-Beta Decay of ${ }^{130}$ Te with CUORE, AHEP 2015, 879871 (2015).

[112] D.R. Artusa et al., First array of enriched $\mathrm{Zn}^{82}$ Se bolometers to search for double beta decay, Eur. Phys. J C 76, 364 (2016).

[113] V. Grigorieva et al., $\mathrm{Li}_{2} \mathrm{MoO}_{4}$ Crystals Grown by Low-Thermal-Gradient Czochralski Technique, J Mat. Sci. Engineering B 7, 63 (2017).

[114] V.N. Shlegel et al., Recent progress in oxide scintillation crystals development by lowthermal gradient Czochralski technique for particle physics experiments, J. Instrum. 12, C08011 (2017).

[115] A.S. Barabash et al., First test of an enriched ${ }^{116} \mathrm{CdWO}_{4}$ scintillating bolometer for neutrinoless double-beta-decay searches, Eur. Phys. J C 76, 487 (2016).

[116] T. Tabarelli de Fatis, Cerenkov emission as a positive tag of double beta decays in bolometric experiments, Eur. Phys. J C 65, 359 (2010).

[117] C. Nones et al., Superconducting Aluminum Layers as Pulse Shape Modifiers: An Innovative Solution to Fight Against Surface Background in Neutrinoless Double Beta Decay Experiments, J Low Temp. Phys. 167, 1029 (2012). 
[118] N. Casali et al., $\mathrm{TeO}_{2}$ bolometers with Cherenkov signal tagging: towards next-generation neutrinoless double-beta decay experiments, Eur. Phys. J C 75, 12 (2015).

[119] K. Schäffner et al., Particle discrimination in $\mathrm{TeO}_{2}$ bolometers using light detectors read out by transition edge sensors, Astropart. Phys. 69, 30 (2015).

[120] E.S. Battistelli et al., CALDER: neutrinoless double-beta decay identification in $\mathrm{TeO}_{2}$ bolometers with kinetic inductance detectors, Eur. Phys. J C 75, 353 (2015).

[121] D.R. Artusa et al., Enriched $\mathrm{TeO}_{2}$ bolometers with active particle discrimination: Towards the CUPID experiment, Phys. Lett. B 767, 321 (2017). 